\title{
ATORES DO SISTEMA DE COMUNICAÇÃO CIENTÍFICA: APONTAMENTOS PARA DISCUSSÃO DE SUAS FUNÇÕES
}

\author{
ACTORS OF THE SCHOLARLY COMMUNICATION \\ SYSTEM: NOTES FOR DISCUSSION OF THEIR ROLES
}

\author{
Marília Augusta de Freitas ${ }^{a}$ \\ Fernando César Lima Leite ${ }^{b}$
}

\begin{abstract}
RESUMO
Introdução: O sistema de comunicação científica, que é subjacente à comunidade científica, é constituído por diversos elementos e processos, entre os quais seus atores e respectivas funções e atividades. É o resultado das funções e atividades pesquisadores, editoras, bibliotecas, agências de fomento e universidades que contribuem para que a produção, compartilhamento e uso do conhecimento de modo que a ciência possa viabilizada. Objetivo: Identificar as funções e atividades desempenhadas pelos principais atores do sistema de comunicação científica (pesquisadores, editoras, bibliotecas, agências de fomento e universidades). Metodologia: Foi realizado amplo levantamento bibliográfico no Google Scholar, Web of Science e Scopus nos meses de junho e julho de 2015. O levantamento desconsiderou limite temporal e tipo de documento. A estratégia de busca foi a combinação dos termos "pesquisadores", "editoras", "bibliotecas", "agências de fomento" e "universidades" combinados com expressões "comunicação científica", "informação científica", "acesso aberto", "sistema de comunicação", "comunicação na ciência". Além das buscas terem sido realizadas em língua portuguesa e inglesa, foram adotados termos sinônimos e flexão singular/plural. Após análise preliminar dos textos recuperados e seleção dos textos relevantes, foi elaborada revisão de literatura norteada pelo objetivo de identificar funções e atividades desempenhadas pelos principais atores do sistema de comunicação científica. A revisão assumiu caráter de narrativa e foi estruturada a partir de cada um dos atores. Resultados: Os resultados são apresentados em forma de revisão de literatura narrativa em que foram definidas e identificadas funções e atividades desempenhadas por cada um dos atores do sistema de comunicação científica contemplados no estudo. Por fim, foi elaborado diagrama que representa graficamente tanto as funções e atividades dos atores do sistema de comunicação científica quanto às interações entre eles.
\end{abstract}

Descritores: Sistema de comunicação científica. Atores do sistema de comunicação

a Doutora em Ciência da Informação pela Universidade de Brasília . Bibliotecária da Universidade de Brasília. E-mail: marilia@bce.unb.br.

b Professor da Faculdade de Ciência da Informação da Universidade de Brasília. E-mail: fernandodfc@gmail.com. 
científica. Funções da comunicação científica.

\section{INTRODUÇÃO}

A comunidade científica caracteriza-se por interesses e práticas em comum, que direcionam os indivíduos no estudo de um determinado problema, sendo fundamental a concepção da ciência aceita pelo grupo. Kuhn (2005, p. 222) afirmou que uma comunidade científica é constituída por praticantes de uma especialidade científica. Em suas palavras, o autor considerou que que tais praticantes foram submetidos a uma iniciação profissional e a uma educação similar, em uma extensão sem paralelos em relação à maioria das outras disciplinas. Para o autor, nesse processo os pesquisadores internalizaram a mesma literatura e dela retiraram muitas vezes as mesmas lições. Normalmente as fronteiras dessa literatura-padrão marcam os limites de um objeto de estudo científico e, em geral, cada comunidade possui um objeto de estudo próprio (KUHN, 2005, p. 222).

No sentido sugerido por Kuhn (2005, p. 222), comunidade científica pode ser entendida como um sistema social que, segundo Storer (1966, p. 29), é definido como "[...] qualquer associação de elementos dinâmicos, reconhecidamente delimitada, os quais são, de alguma forma, interconectados e interdependentes." Storer (1966) considerou que, mais que isso, tais elementos continuam a operar juntos de acordo com certas leis e de maneira que produzam algumas características de total efeito.

O trabalho desenvolvido pelas comunidades científicas é regido por um sistema que envolve interesses múltiplos, além do interesse individual de cada pesquisador. Fourez (1995, p. 93) confirma tal ponto de vista ao afirmar que "a comunidade científica não só goza de reconhecimento interno, como também externo, ou seja: não apenas dentro do próprio grupo, mas também de fora." $\mathrm{Na}$ mesma linha, Le Coadic (2004, p. 33) conceitua comunidades científicas como "redes de organizações e relações sociais formais e informais que desempenham várias funções", para as quais a comunicação é uma das funções predominantes.

Percebe-se, portanto, que a noção de comunidade científica embute a 
noção de comunicação científica. Ou seja, a existência e a dinâmica das comunidades científicas são viabilizadas, entre outros aspectos, por práticas de comunicação. Sobre essa questão, Mueller (2000, p. 22) entende que a totalidade do trabalho intelectual de estudiosos e pesquisadores depende de um intrincado sistema de comunicação, constituído de canais formais e informais, que são apropriados por pesquisadores com duas finalidades fundamentais, a saber, comunicar resultados que obtêm nas pesquisas que realizam e se informarem dos resultados alcançados por outros pesquisadores. De fato, toda atividade de pesquisa envolve práticas de comunicação, como pontuado pela autora. Nesse sentido, em sua dimensão formal, a comunicação resulta em publicações científicas, responsáveis pela materialização de fluxos de informação científica. Nesse sentido, Leite e Costa (2007, p. 93) definem bem essa situação quando afirmam que

O pesquisador, como um dos atores do processo de comunicação, faz uso do sistema de comunicação em diversos momentos, uma vez que, à medida que produz conhecimento, ele necessariamente também o consome. Logo, no início da criação de um novo conhecimento, o esforço de um pesquisador parte daquilo que foi construído anteriormente por outros pesquisadores. Ou seja, no princípio e durante o processo de criação, o pesquisador recorre à literatura de sua especialidade e aos seus pares. Ao fim, divulga os resultados de sua pesquisa por meio dos veículos de comunicação apropriados à sua área de conhecimento. Torna-se evidente, portanto, que um pesquisador utiliza os canais de comunicação em todo o ciclo do conhecimento - desde a sua criação até a sua divulgação -, o que permite afirmar que existe um complexo sistema de comunicação científica que permeia as comunidades científicas e instituições acadêmicas.

Como foi possível perceber, as comunidades científicas são constituídas por diversos elementos e processos, entre os quais seus atores e respectivas funções e atividades. Tais atores, funções e atividades conformam um sistema de comunicação científica, entendido neste artigo como uma espécie de mecanismo complexo - e em muitos aspectos não compreendido - formado de uma diversidade de canais que são profundamente ligados e que estabelecem interações uns com os outros (MIKHAILOV; CHERNYI; GILIAREVSKII, 1984, p. 387). Para o autor, portanto, o sistema deve ser investigado em sua totalidade, uma vez que as diferentes partes que o compõem exercem várias funções e 
suportam cargas desiguais. Porém, todos eles são importantes para o funcionamento normal do sistema e a nenhuma parte deve ser dada preferência sobre as outras.

Considera-se que pesquisadores, editoras, bibliotecas, agências de fomento e universidades constituem os principais atores do sistema de comunicação científica. Isso porque são eles que mais se relacionam e exercem influência sobre as atividades de produção, distribuição e uso da informação científica. Considerando que tais atores são os mais influentes no contexto da comunicação na ciência, o objetivo deste artigo é identificar, com base na literatura, funções e atividades desempenhadas pelos principais atores do sistema de comunicação científica.

\section{PROCEDIMENTOS METODOLÓGICOS}

Partindo do pressuposto de que pesquisadores, editoras, bibliotecas, agências de fomento e universidades constituem os principais atores do sistema de comunicação científica, ou seja, aqueles atores que exercem maior influência nos processos de produção, distribuição e uso da informação científica, foi realizado amplo levantamento bibliográfico no Google Scholar, Web of Science e Scopus nos meses de junho e julho de 2015. O levantamento desconsiderou limite temporal e tipo de documento. A estratégia de busca foi a combinação dos termos "pesquisadores", "editoras", "bibliotecas", "agências de fomento" e "universidades" combinados com expressões "comunicação científica", "informação científica", "acesso aberto", "sistema de comunicação", "comunicação na ciência". Além das buscas terem sido realizadas em língua portuguesa e inglesa, foram adotados termos sinônimos e flexão singular/plural. Após análise preliminar dos textos recuperados e seleção dos textos relevantes, foi elaborada revisão de literatura norteada pelo objetivo de identificar funções e atividades desempenhadas pelos principais atores do sistema de comunicação científica. A revisão assumiu caráter de narrativa e foi estruturada a partir de cada um dos atores. 


\section{FUNÇÕES E ATIVIDADES DOS ATORES DO SISTEMA DE COMUNICAÇÃO CIENTÍFICA}

\subsection{AUTORES-PESQUISADORES}

São os autores-pesquisadores que iniciam o ciclo da comunicação científica no momento que produzem e registram o conhecimento gerado. Nesse ciclo, autores-pesquisadores realizam a pesquisa, elaboram publicações e atuam em colaboração (BJÖRK, 2007). O processo de condução da pesquisa envolve diversas etapas, como é descrito de forma simplificada por Björk (2007), na Figura 1.

Figura 1 - Realização da pesquisa

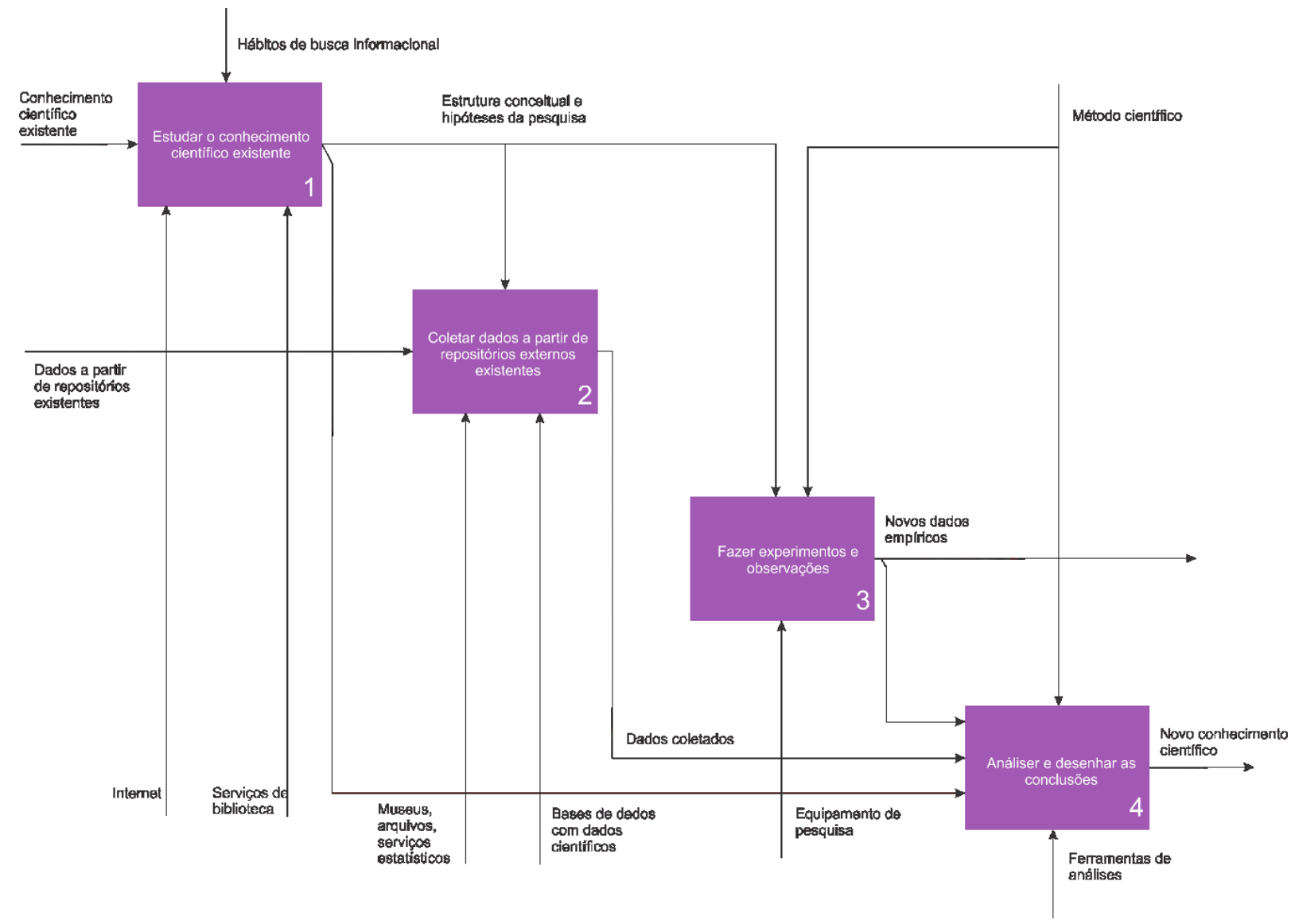

Fonte: Adaptado de Björk (2007, tradução nossa).

De acordo com o Björk, as etapas consecutivas não implicam uma ordem rígida. Assim, o estudo do conhecimento científico existente (atividade 1) pode continuar após o início da segunda e terceira atividades, embora seja sempre a primeira atividade na realização de uma pesquisa (BJÖRK, 2007). Sendo assim, 
o pesquisador, ao mesmo tempo que produz conhecimento, também o consome, estando a etapa de consumo do conhecimento presente em todas as atividades que envolvem a pesquisa. Na mesma linha, Leite e Costa (2007) consideram que

[...] no início da criação de um novo conhecimento, o esforço de um pesquisador parte daquilo que foi construído anteriormente por outros pesquisadores. Ou seja, o pesquisador nunca parte do marco inicial, pois, no princípio e durante o processo de criação, recorre à literatura de sua especialidade e aos seus pares.

Nesse sentido, o diagrama proposto por Björk (2007) (Figura 1) evidencia como pesquisadores conduzem suas pesquisas para a produção de novos conhecimentos científicos. Esses novos conhecimentos gerados constituem uma segunda etapa do processo de comunicação científica, constituída pela comunicação dos resultados. Essa comunicação é essencial para que outros pesquisadores façam uso desses resultados em outros ciclos de produção.

Para comunicar seus resultados de pesquisa, pesquisadores utilizam canais formais e informais. Meadows (2000, p. 25) explica que

Os meios formais são acessíveis de forma fixa por um longo período, e sua aquisição não requer, normalmente, contato com o autor. Livros e periódicos apresentam-se como exemplos típicos deste meio de comunicação. Os canais formais constituem-se de meios de comunicação escritos, quais sejam: livros, periódicos, relatórios, boletins, índices, abstracts.

Os meios formais de comunicação representam as fontes de informação registradas, ou seja, as publicações científicas. Além dos canais formais, existem as fontes não registradas, representadas pelos canais informais. Ambos os canais são utilizados pelos pesquisadores para comunicar suas descobertas (SOUTO, 2003, p. 19), conforme mostra a Figura 2. 
Figura 2 - Sistema de comunicação científica

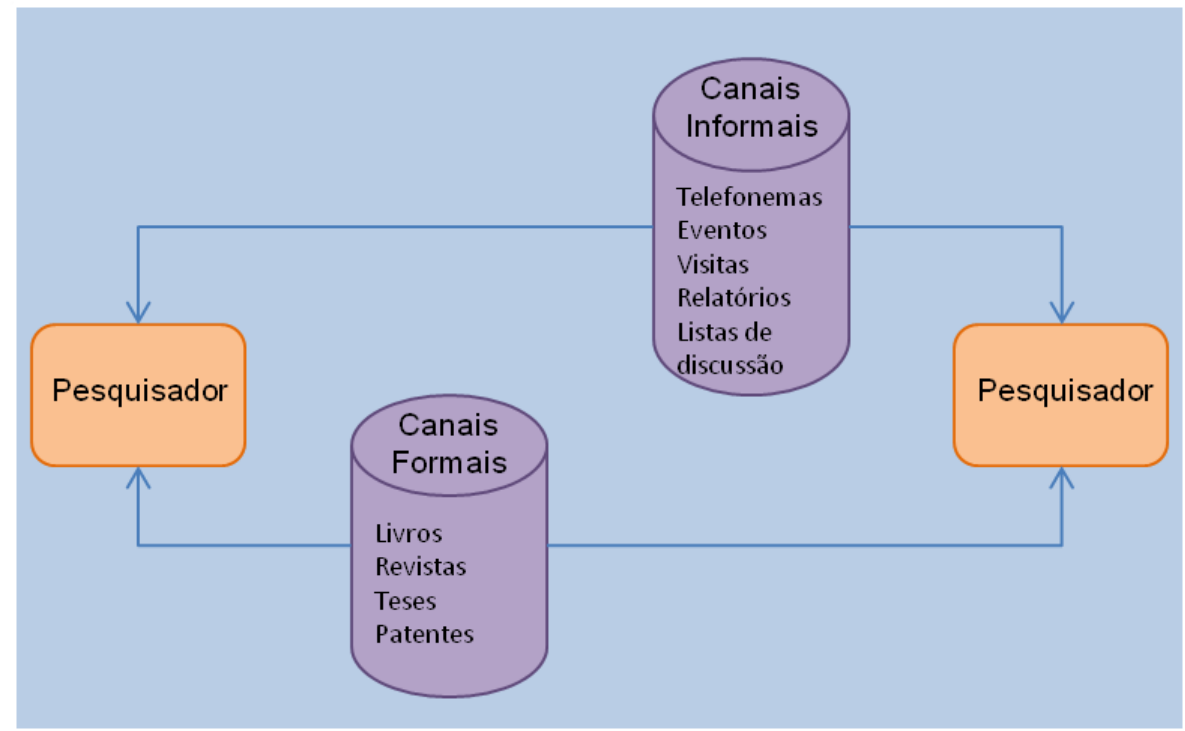

Fonte: Souto (2003, p. 19)

Nesse contexto, o advento das tecnologias proporcionou a criação de modelos alternativos para a comunicação científica, mas a divisão entre canais formais e informais, conforme citado acima, continua pertinente. De acordo com Leite e Costa (2007), "embora, hoje, o desenvolvimento e aplicação de tecnologias no processo de comunicação na ciência tenha tornado o limite entre o formal e o informal cada vez mais nebuloso, essa divisão parece ainda ser pertinente." A mudança mais significativa ocorre nos meios de disseminação, dentro dos canais formais e informais que sofreram mudanças decorrentes do uso das tecnologias, e com a consolidação da internet. Araújo e Freire (1996) afirmam que

[...] na perspectiva dos canais de comunicação da informação, a internet tem dupla função: permite a ligação entre pessoas, de forma livre ou em relação a temas de interesse, ao mesmo tempo em que oferece acesso a documentos, como um serviço de informação ou uma biblioteca fariam.

Nesse cenário inserem-se os repositórios institucionais de acesso aberto, os quais figuram como serviços de informação científica que utilizam a internet para disponibilizar e promover acesso à produção científica de uma instituição. Sendo assim, autores-pesquisadores, enquanto produtores e consumidores do conhecimento científico, exercem um papel primordial para o desenvolvimento do acesso aberto no contexto atual, na medida em que possuem o poder de decidir sobre a forma de disponibilização de seus trabalhos. 
A Bethesda Statement on Open Access Publishing (2003) - uma das principais declarações de acesso aberto - constata que "a publicação eletrônica dos resultados de investigação oferece a oportunidade e a obrigação de compartilhar resultados de pesquisas, ideias e descobertas livremente com a comunidade científica e com o público em geral". Aquela declaração confirma, com convicção, que o pesquisador tem grande interesse em assegurar que os resultados de suas investigações sejam divulgados de imediato, de forma ampla e eficaz.

Pesquisadores têm nos repositórios digitais de acesso aberto um veículo propício para a disseminação de seus resultados de pesquisa e, assim, podem contribuir para a disseminação da informação. Os resultados de pesquisa dizem respeito principalmente aos artigos publicados em periódicos arbitrados, mas também se aplicam a qualquer tipo de pré-publicação não revisada que se pretenda disponibilizar on-line para que seja comentado ou mesmo alertar colegas sobre importantes achados de pesquisa.

Especificamente no contexto dos repositórios institucionais, pesquisadores desempenham um papel fundamental na tomada de decisão pelo depósito de sua produção científica. Isso porque, além de contribuir para a preservação da produção intelectual da instituição, o depósito de trabalhos científicos amplia as chances de serem lidos e citados, promovendo, assim, maior visibilidade e o impacto da pesquisa.

\subsection{EDITORAS}

O papel das editoras científicas é organizar e coordenar todo o processo editorial, que envolve a avaliação e a aprovação do original submetido pelo autor, o contrato, a editoração e a diagramação dos textos, bem como a impressão (nos casos das publicações impressas) ou a disponibilização em meio digital. Tais atividades, em uma análise mais ampla, estão diretamente relacionadas com a condução de processos que culminam com a produção e a qualificação da informação científica.

Meadows (1999, p. 127), debruçando-se sobre as funções das editoras no sistema de comunicação científica, constata que "a atribuição das editoras é 
receber as obras dos autores, organizá-las de forma que sejam aceitáveis para os leitores e, em seguida, divulgá-las." Para Meadows, as editoras envolvidas na produção de livros e periódicos científicos normalmente são as editoras comerciais, as editoras universitárias e outras editoras institucionais, além das sociedades e associações científicas e profissionais.

De modo similar a Meadows (1999, p. 127), o relatório da Wellcome Trust (2004, p.7), uma das maiores fundações dedicadas à pesquisa biomédica do mundo, subdivide as editoras de periódicos acadêmicos em três grupos:

- editoras comerciais, que buscam maximizar o lucro por meio da publicação;

- editoras universitárias, que procuram publicar trabalhos de alta qualidade e, assim, obter lucro simultâneo;

- $\quad$ sociedades científicas, que são instituições sem fins lucrativos, cujos objetivos são geralmente ligados à promoção dos interesses de sua pesquisa ou áreas disciplinares.

Em suma, editores são responsáveis por contribuir tanto para a produção e a qualificação da informação científica quanto para a sua distribuição e circulação nas comunidades científicas. Isso ocorre por meio da comercialização de produtos de informação (venda de livros, assinatura de periódicos científicos ou bases de dados), disseminação na modalidade "acesso aberto" e suas respectivas variações e modelos de negócios.

As atividades relacionadas às editoras contemplam: a organização das publicações, a negociação para assinaturas, o planejamento do periódico (envolvendo questões essenciais, como, por exemplo, a definição do conselho científico), o marketing geral e a definição de preços de assinaturas (BJÖRK, 2007). Essas atividades, bem como a criação e a manutenção de infraestruturas tecnológicas (especialmente no caso de periódicos digitais), geram despesas, que ficam embutidas nos valores cobrados pelas assinaturas. Björk (2007) lembra, porém, que o custo do periódico impresso é maior do que o do eletrônico, visto que o primeiro envolve as atividades de impressão e distribuição. Ainda de acordo com esse autor, a diferença entre o formato em papel e o eletrônico é que a publicação impressa implica um modelo de assinaturas ou pay-per-view, 
enquanto a eletrônica pode fazer uso de uma variedade maior de modelos que gerem receita.

Editoras do mundo inteiro, cientes da importância da difusão do acesso aberto e da adesão crescente da comunidade científica a esse sistema, tentam se adequar a esse novo contexto por meio da adoção, entre outros recursos, de novos modelos de negócios. Foi sob essa influência que surgiu o Article Processing Charge (APC), que vem a ser uma taxa de publicação cobrada aos autores a título de pagamento pela publicação em um periódico acadêmico. Essa taxa costuma ser paga pela instituição ou pelo financiador da pesquisa de um autor. Tratando do APC, Solomon e Björk (2012, p. 1485) explicam que

O modelo APC muda fundamentalmente as relações entre autores, editores e leitores, transferindo a função de financiar a publicação dos assinantes, mais frequentemente bibliotecas universitárias, para os autores, seus financiadores, ou empregadores. O custo de APCs acrescenta uma nova dimensão para as decisões dos autores quanto ao local para tentar publicar seus manuscritos. Também muda o foco dos esforços de marketing dos editores, em que os seus clientes, pelo menos no sentido financeiro, são agora os autores, em vez dos assinantes. Além disso, o papel tradicional das bibliotecas acadêmicas como uma intermediária entre os leitores e editores desaparece, porém, em alguns casos, eles assumiram um novo papel de gerenciar o pagamento dos APCs para os autores em suas universidades.

Com a adoção do modelo APC, o pesquisador fica livre para fazer o uso que lhe aprouver de seu artigo, sendo, por exemplo, possível disponibilizá-lo no repositório de sua instituição ou até mesmo em seu site pessoal. Um estudo de 2015 (SCHIMMER; GESCHUHN; VOGLER, 2015) mostra que a transformação de grande escala do modelo de assinaturas para publicação de acesso aberto é possível e sem despesas adicionais. O estudo foi realizado tendo como base os dados sobre APC da Alemanha, do Reino Unido e da França.

O modelo APC tem sido, porém, criticado porque seus gestores costumam negligenciar o padrão de qualidade das publicações. De acordo com Bell (2012, p. 179), atualmente, há periódicos dispostos a aceitar quase todos os artigos, desde que o autor esteja disposto a pagar a taxa. Autores, em vez das bibliotecas, são os clientes das editoras de acesso aberto, sendo assim um poderoso incentivo para manter a qualidade foi perdido. Esses editores são 
conhecidos como "editores predatórios", os quais, conforme adverte Nassi-Caló (2015),

[...] causam um enorme dano à imagem de periódicos de Acesso Aberto que cobram APC, devido principalmente a artigos sensacionalistas publicados na literatura científica, alegando que Acesso Aberto é sinônimo de publicações de baixa qualidade, metodologia discutível e sem avaliação por pares. Consequentemente, muitos autores relutam em enviar seus artigos a periódicos de acesso aberto, e faz com que seu ingresso em bases renomadas como o Web of Science seja gravemente dificultado.

Por outro lado Björk e Solomon (2014, p. 22) equilibram a questão quando lembram que periódicos editados por "editores predatórios" dificilmente serão indexados em bases referenciais conceituadas, como a Scopus ${ }^{1}$. Os autores afirmam que

[...] esses periódicos são, portanto, pouco prováveis de serem escolhidos como fonte de publicação por autores que recebem financiamento do governo ou de agências de fomento, pelo menos em países com liderança acadêmica, como o Reino Unido.

Discussões à parte, esse modelo tem se apresentado como uma forma sustentável para os periódicos científicos no cenário do acesso aberto. De acordo com Björk (2013, p. 13), "o modelo de negócios baseado na APC para a publicação de periódicos de acesso aberto provou a sua sustentabilidade, pelo menos nas publicações da área biomédica."

Nesse sentido, os repositórios institucionais beneficiam-se desses modelos na medida em que podem disponibilizar, coletar e organizar essa produção institucional disponível em acesso aberto.

\subsection{BIBLIOTECAS UNIVERSITÁRIAS/BIBLIOTECÁRIOS}

Outrora, o papel da biblioteca universitária restringia-se a adquirir informações por meio da aquisição de livros e periódicos, e torná-los disponíveis e acessíveis aos usuários. De acordo com Cunha (2000, p. 73)

[...] através dos séculos, o ponto focal da universidade tem sido a biblioteca, com o seu acervo de obras impressas preservando

\footnotetext{
${ }^{1}$ Scopus é um banco de dados de resumos e citações de artigos de periódicos acadêmicos. Possui em torno de 19 mil títulos, de cerca de 5 mil editoras. É de propriedade da Elsevier e está disponível na internet, mediante assinatura.
} 
o conhecimento da civilização. Atualmente, esse conhecimento existe sob muitas formas: texto, gráfico, som, algoritmo e simulação da realidade virtual e, ao mesmo, ele existe literalmente no éter, isto é, distribuído em redes mundiais, em representações digitais, acessíveis a qualquer indivíduo e, com certeza, não mais uma prerrogativa de poucos privilegiados da academia. (CUNHA, 2000, p. 73).

No contexto da comunicação científica, as bibliotecas universitárias assumem o papel de facilitadoras da disseminação e da recuperação da informação. Para Meadows (1999, p. 127), "bibliotecários são responsáveis por codificar e armazenar o material oriundo das editoras de modo a torná-lo acessível aos leitores." Sendo assim, o papel da biblioteca universitária é gerir a informação, contribuindo substancialmente para a produção do conhecimento.

A biblioteca universitária, em especial, tem como principal missão garantir e promover o acesso à informação científica, contemplando as atividades de ensino, pesquisa e extensão. De acordo com Ohira (1998)

Para a biblioteca universitária o apoio acadêmico já é tradicional
e seu papel vem sendo cumprido, ainda que às custas (sic) de
esforços, considerando as condições por que passa a maioria
das bibliotecas universitárias brasileiras. No apoio à pesquisa,
vem se firmando e cada vez mais ocupando lugar de destaque
nas universidades, necessitando ainda de estruturas
informacionais adequadamente capazes de atender, de forma
satisfatória às necessidades dos pesquisadores e gerar
informações indispensáveis ao fomento das atividades de
pesquisa.

A forma como no passado as bibliotecas universitárias garantiam e promoviam o acesso à informação científica sofreu mudanças significativas com os avanços tecnológicos, obrigando os bibliotecários a se adaptar a elas. O uso das tecnologias de informação passou a constituir elemento essencial na oferta de serviços de informação a fim de garantir a ampliação do acesso à informação.

Nesse cenário, em que a internet constitui um dos instrumentos de maior impacto no acesso à informação científica, bibliotecas universitárias têm se valido de duas estratégias: fornecer acesso por meio do acesso aberto - na forma de manuscritos ou cópias de publicações formais em periódicos, disponibilizadas em repositórios digitais de acesso aberto - ou por meio de assinatura de bases de dados. No caso das bases de dados, o acesso é dado por fornecedores que vendem seus serviços de informação às bibliotecas, cujo 
objetivo é centralizar o processo de busca e recuperação dessa informação (BJÖRK, 2007).

No caso específico do acesso aberto, de acordo com Costa (2005, p. 176177), os repositórios institucionais provocam a entrada das bibliotecas em cena. Para a autora, repositórios são centrados na preocupação com a acessibilidade ampla e irrestrita à informação, resultado do movimento recente em direção à filosofia aberta para os arquivos em que os conteúdos gerados por pesquisadores estão disponíveis.

Nesse sentido, bibliotecários assumem um papel de disseminadores da produção científica de instituições acadêmicas, porque são eles, na maioria dos casos, os responsáveis pela gestão dos repositórios institucionais.

Leite (2009, p. 38) mostra que "os processos envolvidos nas rotinas de um repositório institucional possuem natureza muito próxima e similar aos trabalhos desenvolvidos em ambientes digitais por bibliotecas e bibliotecários." Segundo o autor, são os bibliotecários que possuem expertise para a elaboração de políticas de formação, desenvolvimento e gestão de coleções, além de serem as bibliotecas a instância organizacional mais ligada às questões de comunicação e gestão da informação científica.

É função do bibliotecário, na condição de mediador entre o pesquisador e os leitores, gerenciar os repositórios institucionais e o desenvolvimento de suas coleções. Esse desenvolvimento pode se dar por meio do depósito mediado, no qual o próprio bibliotecário se responsabiliza pela inclusão de itens ou pelo autoarquivamento. Esse segundo procedimento, por mais que seja realizado e incentivado por parte das universidades, não dispensa o papel do bibliotecário na gestão e na manutenção dos repositórios institucionais.

A Bethesda Statement on Open Access Publishing (2003) propõe que bibliotecários e bibliotecas assumam peremptoriamente o papel de desenvolver e apoiar mecanismos para a transição das publicações para o acesso aberto, e divulgar esses mecanismos para a comunidade. Ademais, também cabe à biblioteca ensinar aos usuários os benefícios da publicação em acesso aberto, além de destacar as revistas disponíveis nessa modalidade.

Segundo Antelman (2004, p. 374), os bibliotecários que optarem por 
implementar repositórios institucionais

[...] devem ser capazes de convencer os professores, muitos dos quais são, por várias razões, relutantes em compartilhar sua produção de pesquisa principal. Dados mostram, no entanto, que artigos disponíveis gratuitamente, pela maior facilidade de uso, são mais propensos a serem citados, o que é uma poderosa evidência do valor dos repositórios, bem como de outros canais de acesso aberto.

Confirma-se aqui, mais uma vez, a importância da atuação de bibliotecários e bibliotecas na viabilização do acesso aberto, principalmente os profissionais das universidades, local onde é, aliás, desenvolvida a maior parte das pesquisas científicas (BAPTISTA et al., 2007). Sua atuação é fundamental tanto para decidir sobre questões gerenciais associadas ao acesso aberto, quanto políticas ligadas à inclusão de conteúdos.

\subsection{AgÊNCIAS DE FOMENTO}

Em todo o mundo, boa parte da ciência e dos projetos de pesquisa é financiada por agências governamentais de fomento. No Brasil, como grande parte das pesquisas científicas é desenvolvida no âmbito da Pós-Graduação stricto sensu em universidades públicas, pode-se dizer que as agências de fomento são também as principais responsáveis pelo financiamento, uma vez que os programas de pós-graduação são financiados pela Coordenação de Aperfeiçoamento de Pessoal de Nível Superior (Capes). Silva e Ferro (2010, p. 2) recordam que

[...] desde a implantação dos programas de pós-graduação stricto sensu no Brasil até o momento atual de expansão de tais programas, existe a necessidade não apenas de políticas educacionais para proporcionar a implantação, consolidação e expansão da pós-graduação stricto sensu, mas também do aporte de recursos financeiros a serem utilizados para oferecer estes programas com qualidade à sociedade brasileira. Os recursos financeiros contribuem para proporcionar a excelência dos programas de pós-graduação, sendo em grande medida provenientes das agências de fomento.

À título de exemplificação, para fomentar a pesquisa científica no âmbito de universidades e instituições de pesquisa, governo brasileiro mantém agências financiadoras da produção do conhecimento científico e tecnológico. É o caso do Conselho Nacional de Desenvolvimento Científico e Tecnológico (CNPq), da 
Capes e da Financiadora de Estudos e Projetos (Finep), entre outras agências federais, além das Fundações de Amparo à Pesquisa (FAPs) estaduais, que estão presentes em 21 das 27 unidades federativas.

Agências de fomento influenciam sobremaneira 0 processo de comunicação científica no sentido atribuído por Garvey (1979), que considera que o processo de comunicação tem a ver com a produção, a distribuição e o uso da informação. As agências atuam na produção, porquanto financiam a atividade de pesquisa; na distribuição, já que, para concederem financiamento, precisam avaliar o pesquisador por meio de sua produção científica; e no uso, considerando que a maior ou menor valorização de determinados canais de comunicação influencia no maior ou no menor uso deles por parte de outros pesquisadores.

Segundo Souza (2013, p. 53), agências de fomento dispõem configurados como objetivos, missões ou dispositivos institucionais quanto ao seu papel na difusão científica - de um cabedal de: a) políticas, planos ou programas voltados à garantia e à ampliação do acesso à informação, tanto sobre pesquisas financiadas quanto sobre seus resultados; b) produção de diversas publicações, disponibilizadas na internet, para auxiliar na sensibilização e no desenvolvimento de interesses e habilidades em comunicação científica; e c) ações voltadas para o contato ou o intercâmbio de informações das agências com diversos setores (universidades, pesquisadores, empresas, setores do governo, entre outros).

Ainda de acordo com a autora, no Brasil, as agências, além de responderem pelo forte crescimento das pesquisas científicas nacionais e pela consolidação da pós-graduação stricto sensu, desempenham importante papel na comunicação das informações científicas, com a finalidade de veicular as pesquisas apoiadas (SOUZA, 2013).

Nesse sentido, às agências de fomento cabe grande parte da responsabilidade pelo necessário avanço do acesso aberto e pelo desenvolvimento de repositórios institucionais em universidades e instituições de pesquisa, na medida em que os repositórios podem ser utilizados como mecanismo para a disseminação de produção científica. 
O Manifesto Brasileiro de Apoio ao Acesso Livre à Informação Científica (IBICT, 2005) lembra, por sua vez, que é necessário que as agências de fomento reconheçam a publicação científica em repositórios de acesso aberto para efeito de avaliação da produção científica dos pesquisadores e para a concessão de auxílio e financiamento para a pesquisa, além de recomendar que os pesquisadores que recebam auxílio depositem seus trabalhos nesses repositórios. Ademais, o manifesto incentiva essas agências a promover e a apoiar a construção e a manutenção de repositórios institucionais e temáticos, bem como a dar suporte prioritariamente à edição de publicações científicas eletrônicas de acesso aberto.

Esse manifesto (IBICT, 2005) segue a mesma linha de conduta da Bethesda Statement on Open Access Publishing (2003), ao sugerir que as agências de fomento incentivem pesquisadores a depositar seus trabalhos de acordo com os princípios do acesso aberto, maximizando, desse modo, o acesso e beneficiando outros cientistas, estudiosos e o público em geral.

Um dos primeiros resultados da regulamentação daqueles propósitos nasceu com a política adotada pela National Institutes of Health $(\mathrm{NIH})$, nos Estados Unidos, em fevereiro de 2005, que se tornou efetiva em maio do mesmo ano. Essa política incentiva os pesquisadores que fazem uso de bolsas de estudo a depositar suas publicações no PubMed Central (PMC), no prazo de 12 meses, a contar da data de publicação. Em 2008, essa política foi revista, tendo substituído o incentivo ao depósito por obrigação de depósito.

Seguindo o mesmo modelo, a Wellcome Trust modificou suas condições de concessão e tornou obrigatório, a partir de outubro de 2006, que seus trabalhos financiados estivessem em acesso aberto via PMC, no prazo máximo de seis meses da publicação.

No Reino Unido, as agências de fomento criaram políticas de acesso aberto para pesquisas financiadas com dinheiro público. De acordo com Pinfield (2010, p. 40), "a maior parte dos conselhos de pesquisa adotou políticas de acesso aberto em 2006, como parte de uma iniciativa de articulação dos Reserach Councils UK (RCUK)." Os conselhos remanescentes adotaram essas políticas posteriormente: Arts and Humanities Research Council (AHRC), em 
setembro de 2007, e Engineering and Physical Sciences Research Council (EPSRC), em janeiro de 2009.

Outros países, a exemplo da Austrália, da Áustria, da Bélgica, do Canadá, da França, da Alemanha, da Irlanda, da Itália, da Espanha e da Suíça, também adotaram políticas semelhantes à do Reino Unido, determinando que pesquisas financiadas pelo governo fossem disponibilizadas em acesso aberto.

No Brasil, não foi identificada, na literatura, nenhuma política de acesso aberto formulada por agências de fomento, nos moldes das citadas. Uma pesquisa elaborada por Costa (2014, p. 177-178) demonstrou que certas agências de fomento, como a Fapesp e Fapemig, possuem, em suas bibliotecas virtuais, informações e/ou relatórios de seus projetos financiados. Entretanto, esse sistema não se configura como um repositório no contexto do acesso aberto, pois apresenta apenas informações referenciais das pesquisas, não disponibilizando, por exemplo, o texto completo, ou seja, aquilo que foi aceito pelos pares.

Por fim, segundo Costa (2014, p. 180), a América Latina não parece acompanhar a tendência de algumas regiões como a Europa e América do Norte que têm se mobilizado em discussões sobre como tornar efetiva as políticas de suas agências para a promoção do acesso do conhecimento científico produzido com seus recursos.

\subsection{UNIVERSIDADES}

Considerando a produção, a distribuição e o uso da informação como processos do sistema de comunicação científica, definidos por Garvey (1979), a universidades atuam diretamente nos três processos citados. De acordo com Leite e Costa (2007),

[...] as universidades, como comunidades acadêmicas, constituem elementos do sistema científico. São consideradas ainda como o cerne da produção do conhecimento, e os processos de comunicação científica permeiam boa parte de suas atividades, o que permite tanto as trocas internas de conhecimento quanto externas, em interação com comunidades científicas.

No contexto brasileiro, as universidades públicas constituem o principal 
suporte institucional para a pesquisa e para a formação de pesquisadores (DURHAM, 1998). Assim, no âmbito do sistema de comunicação científica, as universidades são responsáveis pelas infraestruturas organizacional, tecnológica e política, no que concerne às atividades de pesquisa, bem como pelo compartilhamento do conhecimento e das experiências resultantes das pesquisas científicas.

Na mesma linha, considerando a comunicação científica, Leite (2006, p. 216) entende que as universidades influenciam os processos de criação e compartilhamento do conhecimento, na medida em que subsidiam a participação em eventos científicos e oferecem infraestrutura tanto para a pesquisa quanto para o ensino. A produção do conhecimento em universidades está representada na Figura 3.

Figura 3 - Produção do conhecimento em universidades

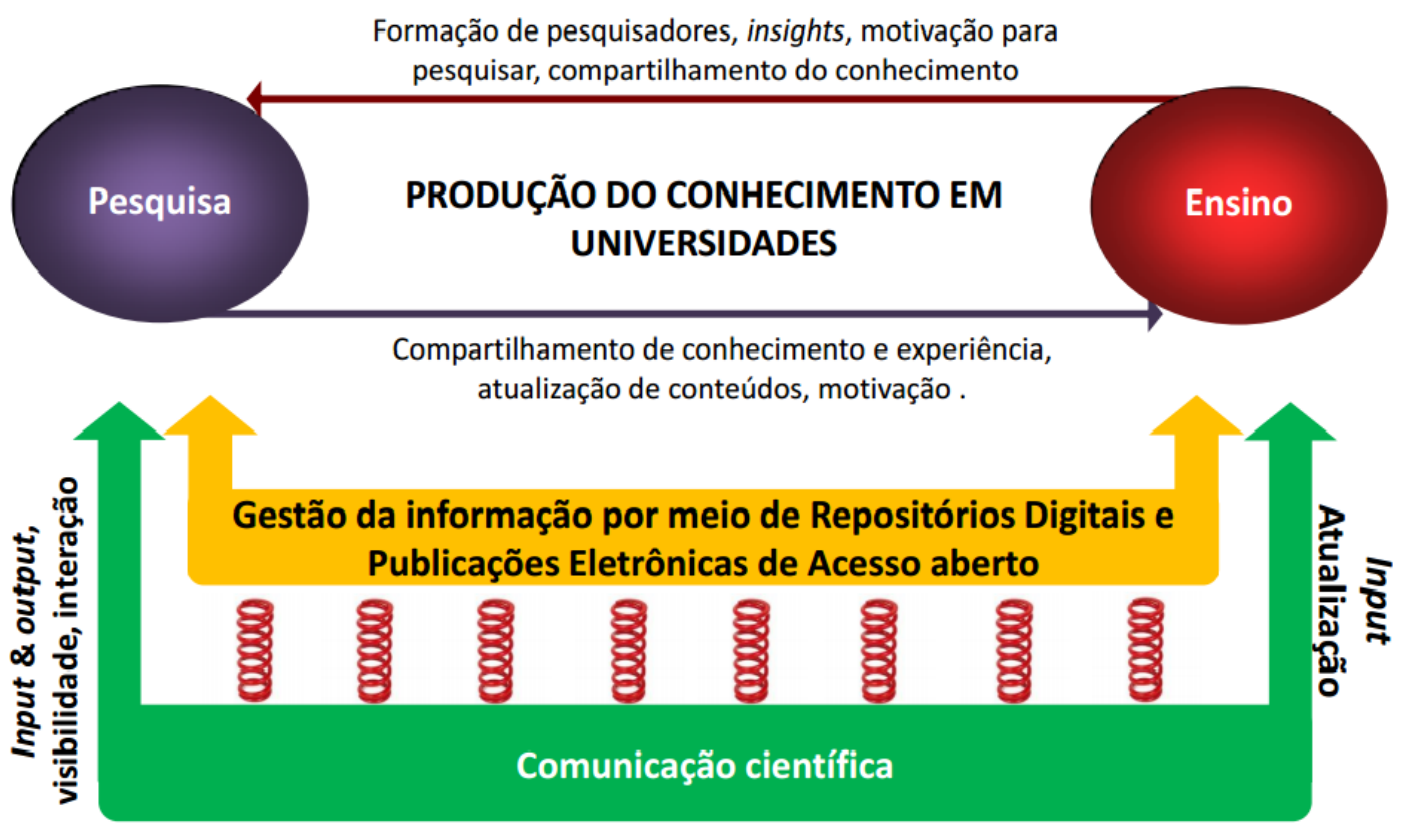

Fonte: Leite (2014).

Analisando a Figura 3, é possível concluir que o compartilhamento de conhecimentos e experiências é o elemento condutor da pesquisa e do ensino, assim como é uma das funções do sistema de comunicação científica global (BJÖRK, 2007). Esse compartilhamento está relacionado diretamente à gestão da informação, que, no cenário atual, tem, nos repositórios institucionais de acesso aberto, eficientes canais e poderosas ferramentas para a gestão do 
conhecimento. No âmbito das universidades, porém, para que a prática do acesso aberto seja viabilizada, é essencial que elas definam políticas que norteiem as ações de pesquisadores, bibliotecários e de suas editoras.

O Manifesto Brasileiro de Apoio ao Acesso Livre à Informação Científica (IBICT, 2005) recomenda que as universidades encorajem os pesquisadores a publicar seus resultados de pesquisa em periódicos de acesso aberto, e a depositar seus trabalhos no repositório institucional de suas instituições. Para que isso se concretize, as instituições acadêmicas devem valorizar a publicação em ambiente de acesso aberto no sistema de avaliação de mérito e progressão salarial.

A disponibilização da produção científica em acesso aberto é vantajosa para a instituição acadêmica na medida em que promove e maximiza a disseminação da sua produção científica. Um estudo de Antelman (2004) mostra que artigos em acesso aberto têm um impacto bem maior sobre uma variedade de áreas do conhecimento científico do que aqueles que não estão disponíveis gratuitamente.

Além dos estudos de Antelman (2004), outros demonstram que publicações disponíveis em acesso aberto tendem a ser mais citadas e, por consequência, podem trazer maior visibilidade para o pesquisador e sua instituição (LAWRENCE, 2001; HARNARD, BRODY, 2004; KURTZ et al., 2004; BRODY et al., 2004; EBRAHIM et al., 2014).

Em suma, universidades devem fomentar políticas que viabilizem a disseminação de suas produções científicas em acesso aberto. A adoção de uma política de depósito compulsório da produção científica em um repositório institucional de acesso aberto é uma medida que pode ser adotada pelas instituições para maximizar o impacto dos resultados de pesquisas.

Gargouri et al. (2012) confirmam que "[...] políticas de depósito compulsório em repositórios institucionais de acesso aberto têm um grande efeito, e quanto mais forte o mandato, mais forte será o efeito." A título de exemplo, cita-se a política adotada pela Universidade de Liège (na Bélgica) ${ }^{2}$, que atrela a progressão na carreira às publicações que seus pesquisadores têm no

2 http://recteur.blogs.ulg.ac.be/?p=103 
repositório institucional. No Brasil, a falta de estrutura é uma das principais limitações para a consolidação dos repositórios institucionais. De acordo com Weitzel e Machado (2010, p. 6),

[...] nem todas as instituições de ensino e pesquisa no Brasil possuem condições técnicas, operacionais, humanas e materiais para instalar um software (para criação de repositórios digitais) em um servidor e desenvolver um repositório digital.

Com efeito, o desenvolvimento de repositórios institucionais depende de um trabalho conjunto por parte da comunidade acadêmica, visando garantir o acesso à produção científica da instituição.

\section{CONCLUSÕES}

A discussão acerca das funções e atividades desempenhadas pelos principais atores do sistema de comunicação científica realizada com base na literatura pertinente, permite conclusão do artigo com o apontamento das atribuições fundamentais de cada um deles. Nesse sentido, autorespesquisadores são responsáveis pela produção, pelo registro e pelo consumo de conhecimento. Sofrem influências de todos os demais atores no processo de condução da pesquisa. Editores, por seu turno, são responsáveis pela validação, pela certificação, pela publicação e pela distribuição da pesquisa. Esta última pode se dar por meio do acesso aberto ou do acesso pago. Os principais clientes do acesso pago são as bibliotecas universitárias, responsáveis pelas assinaturas. Já para o acesso aberto, o principal cliente é o usuário final, ou seja, o próprio autor-pesquisador.

Por outro lado, e de modo complementar, bibliotecas universitárias são responsáveis pela gestão da informação. Essas bibliotecas provêm serviços de informação com a finalidade de garantir acesso à informação científica para a condução de pesquisas. Esse acesso pode ser feito por meio de bases de dados, de portais de periódicos de acesso restrito e acesso aberto, e também por meio de repositórios digitais. As universidades, como lócus da produção do conhecimento, são responsáveis por garantir infraestrutura científica e tecnológica para a realização de pesquisas e, além disso, por gerirem pesquisas científicas realizadas no âmbito da instituição e por estabelecerem políticas 
institucionais. Por fim, agências de fomento são responsáveis pelo financiamento de pesquisas científicas, também interferem diretamente na gestão da informação, na medida em que financiam o acesso a periódicos pagos. Por consequência, interferem diretamente na produção e no consumo do conhecimento. São também responsáveis pelas políticas científicas, que regulamentam os cursos de Pós-Graduação stricto sensu em universidades públicas, onde é produzida grande parte das pesquisas científicas.

Por fim, as funções dos atores que participam do sistema de comunicação científica discutidas ao longo do artigo estão representadas graficamente na Figura 4.

Figura 4 - Funções dos atores que participam do sistema de comunicação científica

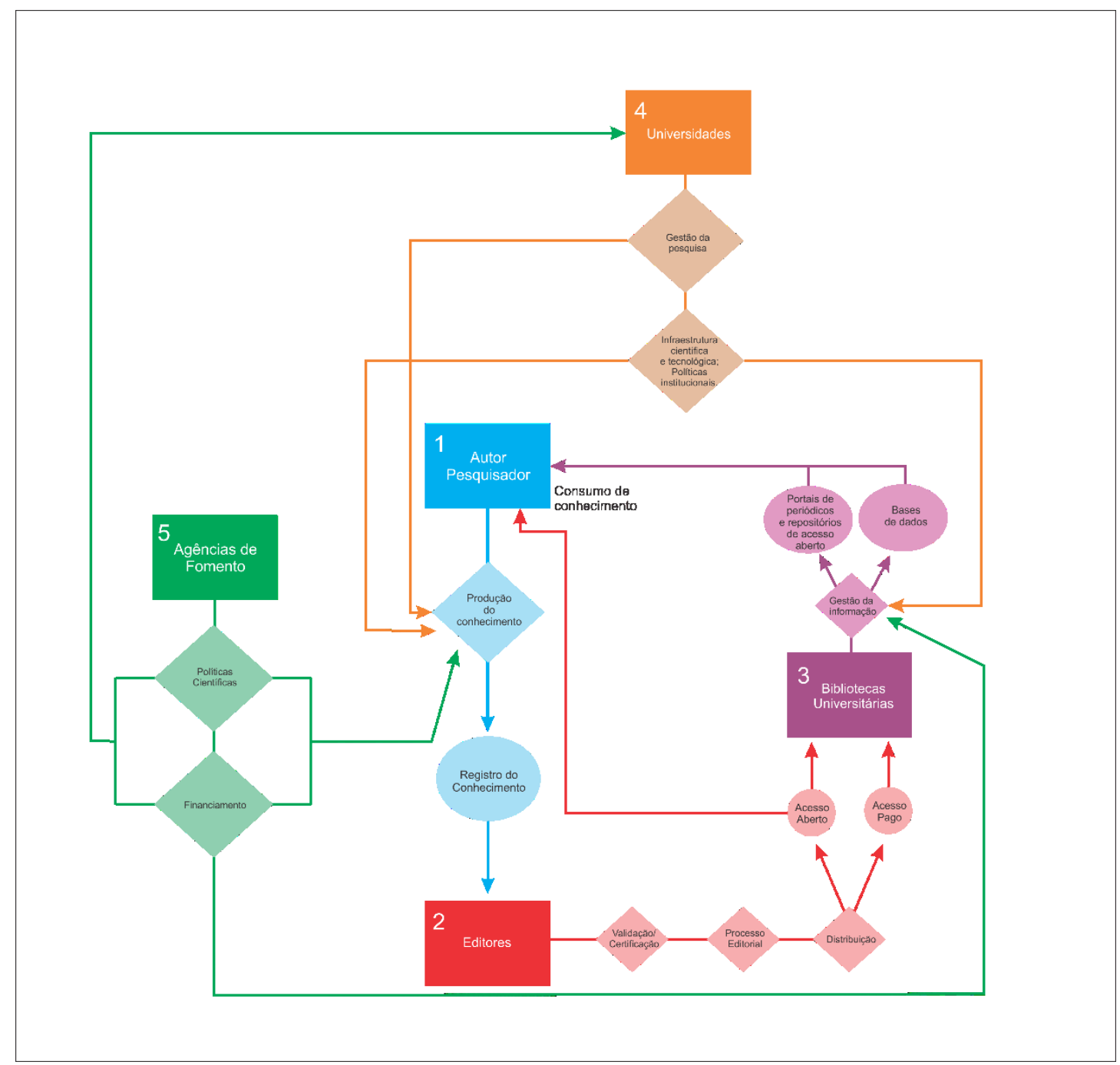

Fonte: Elaboração própria. 


\section{REFERÊNCIAS}

ANTELMAN, K. Do open-access articles have a greater research impact? College \& Research Libraries, Chicago, v. 65, n. 5, 2004, p. 372-382.

ARAÚJO, V. M. R. H. de; FREIRE, I. M. A rede internet como canal de comunicação, na perspectiva da Ciência da Informação. Temas em Debate, v. 8, n. 2, maio/ago. 1996. Disponível em:

http://www.isafreire.pro.br/ArtigoAREDEINTERNET.htm. Acesso em: 08 dez. 2015.

BAPTISTA, A. A.; COSTA, S. M. de S.; KURAMOTO, H.; RODRIGUES, E. Comunicação científica: o papel da Open Archives Initiative no contexto do Acesso Livre. Encontros Bibli: Revista Eletrônica de Biblioteconomia e Ciência da Informação, Florianópolis, n. esp., 2007. Disponível em: https://periodicos.ufsc.br/index.php/eb/article/viewFile/15182924.2007v12nesp1 p1/435. Acesso em: 08 dez. 2015.

BETHESDA Statement on Open Access Publishing. 2003. Disponível em: http://legacy.earlham.edu/ peters/fos/bethesda.htm. Acesso em: 11 fev. 2013

BELL, J. Predatory publishers are corrupting open access. Nature, v. 489, set. 2012. Disponível em:

http://www.nature.com/polopoly_fs/1.11385!/menu/main/topColumns/topLeftCol umn/pdf/489179a.pdf. Acesso em: 01 dez. 2015.

BJÖRK, Bo-Christer. Open Access: are the barriers to change receding?

Publications, v. 1, n.1, p. 5-15, abr. 2013. Disponível em:

http://10.3390/publications1010005. Acesso em: 01 dez. 2015.

BJÖRK, Bo-Christer. A model of scientifitc communication of a global distributed information system. In: PROCEEDINGS OF THE IATUL CONFERENCES, 2007.

\section{BJÖRK, Bo-Christer; SOLOMON, D. Developing an effective market for} open access Articles Processing Charges. Wellcome Trust: Londres, 2014. 69 p. Disponível em:

http://www.wellcome.ac.uk/stellent/groups/corporatesite/@policy_communicatio ns/documents/web_document/wtp055910.pdf. Acesso em: 01 dez. 2015.

BRODY, T.; STAMERJOHANNS, H.; VALLIĖRES, F.; HARNAD, S.; GINGRAS, Y.; OPPENHEIM, C. The Effect of Open Access on Citation Impact. lam, 2004. Disponível em: http://opcit.eprints.org/feb19oa/brody-impact.pdf. Acesso em: 12 fev. 2014.

COSTA, S. M. S. A comunicação científica nos dias atuais: impactos de uma filosofia aberta. Palestra realizada durante a $57^{a}$ Reunião Anual da SBPC. 2005. Disponível em: 
http://www.sbpcnet.org.br/livro/57ra/programas/CONF_SIMP/textos/selycosta.ht m. Acesso em: 08 jul. 2013.

COSTA, M. P. da. Características e contribuições da via verde para o Acesso Aberto à informação científica na América Latina. 2014. 226 f. Dissertação (Mestrado em Ciência da Informação) - Universidade de Brasília, Brasília, 2014.

CUNHA, M. B. da. Construindo o futuro: a biblioteca universitária brasileira em 2010. Ciência da Informação, Brasília, v. 29, n. 1, p. 71-89, jan./abr. 2000.

DURHAM, E. R. As universidades públicas e a pesquisa no Brasil. Núcleo de Pesquisas sobre Ensino Superior (NUPES), São Paulo, v. 9, p. 1-28, 1998.

EBRAHIM, N. A.; SALEHI, H.; EMBI, M. A.; HABIBI, F.; GHOLIZADEH, H.; MOTAHAR, S. M. Visibility and Citation Impact. International Education Studies, v. 7, n. 4, p. 120-125, 2014. Disponível em:

http://papers.ssrn.com/sol3/papers.cfm?abstract_id=2419315. Acesso em: 26 nov. 2015.

FOUREZ, G. A construção das ciências: introdução à filosofia e à ética das ciências. São Paulo: Editora da Universidade Estadual Paulista, 1995.

GARGOURI, Y.; LARIVIĖRE, V.; GINGRAS, Y.; CARR, L.; HARNAD, S. Green and Gold Open Access Percentages and Growth, by Discipline. In:

INTERNATIONAL CONFERENCE ON SCIENCE AND TECHNOLOGY INDICATORS (STI), 17., 2012, Montreal. Anais eletrônicos...Montreal: Science and Technology Indicators, 2012. Disponível em: http://journals.plos.org/plosone/article?id=10.1371/journal.pone.0013636. Acesso em: 01 dez. 2015.

GARVEY, W. D. Communication: the essence of Science. Oxford: Pergamon Press, 1979.

HARNARD, S.; BRODY, T. Comparing the Impact of Open Access (OA) vs. Non-OA Articles in the Same Journals. D-Lib magazine, v. 10, n. 6, jun. 2004. Disponível em: http://www.dlib.org/dlib/june04/harnad/06harnad.html. Acesso em: 12 fev. 2014.

INSTITUTO BRASILEIRO DE INFORMAÇÃO EM CIÊNCIA E TECNOLOGIA (IBICT). Manifesto brasileiro de apoio ao acesso livre à informação cientifica. 2005. Disponível em: http://livroaberto.ibict.br/docs/Manifesto.pdf. Acesso em: 08 jul. 2013.

KUHN, T. S. A estrutura das revoluções cientificas. 9. ed. São Paulo: Perspectiva, 2005. 260 p.

KURTZ, M. J.; EICHHORN, G.; ACCOMAZZI, A.; GRANT, C.; DEMLEITNER, M.; MURRAY, S. S. Worldwide Use and Impact of the NASA Astrophysics Data System Digital Library. Journal of the American Society for Information 
Science and Technology, jan. 2004. Disponível em:

https://www.cfa.harvard.edu/ kurtz/jasist1.pdf. Acesso em: 12 fev. 2014.

LAWRENCE, S. Free online availability substantially increases a paper's impact. Nature, v. 411, maio 2001. Disponível em: http://www.hydrology-andearth-system-sciences.net/pr_nature_free_online_availability.pdf. Acesso em: 12 fev. 2014.

LE COADIC, Yves-Françoise. A ciência da Informação. Brasília, DF: Briquet de Lemos, 2004.

LEITE, F. C. L. Acesso aberto e repositórios institucionais: repensando a comunicação e a gestão da informação científica. Belém, 2014. 51 slides, cor. Disponível em: http://bc.ufpa.br/evento/apresentacoes/fernandoLeite.pdf. Acesso em: 08 dez. 2015.

LEITE, F. C. L. Como gerenciar e ampliar a visibilidade da informação científica brasileira: repositórios institucionais de Acesso Aberto. Brasília: IBICT, 2009. 124 p. Disponível em: http://repositorio.unb.br/handle/10482/4841. Acesso em: 10 abr. 2013.

LEITE, F. C. L. Gestão do conhecimento científico no contexto acadêmico: proposta de um modelo conceitual. 2006. 240 f. Dissertação (Mestrado em Ciência da Informação) - Universidade de Brasília, Brasília, 2006. Disponível em: http://repositorio.unb.br/handle/10482/3975. Acesso em: 08 dez. 2015.

LEITE, F. C. L.; COSTA, S. Gestão do conhecimento científico: proposta de um modelo conceitual com base em processos de comunicação científica. Ciência da Informação, Brasília, v. 36, n. 1, jan./abr. 2007. Disponível em: http://www.SciELO.br/SciELO.php?pid=S010019652007000100007\&script=sci_arttext. Acesso em: 26 fev. 2013.

MEADOWS, A. J. Avaliando o desenvolvimento da comunicação eletrônica. In: MUELLER, S. P. M.; PASSO, E. J. L. (Org.). Comunicação científica. Brasília: Universidade de Brasília, 2000.

MEADOWS, A. J. A comunicação científica. Brasília: Briquet de Lemos, 1999.

MIKHAILOV, A. I.; CHERNYI, A. L.; GILIAREVSKII, R. S. Scientific communications and informatics. Arlington: Information Resources, 1984. 402 p.

MUELLER, S. P. M. A ciência, o sistema de comunicação científica e a literatura científica. In: CAMPELLO, B. S.; CENDÓN, B. V.; KREMER, J. M. (Org.). Fontes de informação para pesquisadores e profissionais. Belo Horizonte: Editora UFMG, 2000.

NASSI-CALÓ, L. Acesso Aberto na América Latina livre de periódicos predatórios. Scielo em perspectiva, nov. 2015. Disponível em: 
http://blog.scielo.org/blog/2015/11/13/periodicos-predatorios-o-lado-obscuro-doacesso-aberto/. Acesso em: $01 \mathrm{dez} .2015$.

OHIRA, M. L. B. Porque fazer pesquisa na universidade? Revista ABC, v. 3, n. 3, 1998. Disponível em: http://revista.acbsc.org.br/racb/article/view/329/388. Acesso em: 08 dez. 2015.

PINFIELD, S. Paying for open access? Institutional funding streams and OA publication charges. Learned Publishing, v. 23, n. 1, p. 39-52, jan. 2010.

SCHIMMER, R.; GESCHUHN, K. K.; VOGLER, A. Disrupting the subscription journals' business model for the necessary large-scale transformation to open access. Max Planck Digital Library, 2015. Disponível em: http://10.17617/1.3. Acesso em: 03 dez. 2015.

SILVA, S. M. V. da; FERRO, M. do A. B. CAPES e CNPq: agências de fomento e desenvolvimento para a pós-graduação brasileira. In: ENCONTRO DE PESQUISA EM EDUCAÇÃO, 6., 2010, Teresina. Anais eletrônicos...Teresina: 2010. Disponível em:

http://www.ufpi.br/subsiteFiles/ppged/arquivos/files/VI.encontro.2010/GT.10/GT _10_06_2010.pdf. Acesso em: 14 set. 2013.

SOLOMON, D. J.; BJÖRK, Bo-Christer. A Study of Open Access Journals Using Article Processing Charges. Journal of the American Society for Information Science and Technology (JASIST), v. 63, n. 8, p. 1485-1495, 2012.

SOUTO, L. F. O leitor universitário e sua formação quanto ao uso de recursos informacionais. In: CONGRESSO BRASILEIRO DE LEITURA, 14., 2003, Campinas. Anais... Campinas, 2003. Disponível em: http://eprints.rclis.org/5456/1/2004_003.pdf. Acesso em: 08 dez. 2015

SOUZA, M. B. C. P. de. A difusão científica em agências governamentais de fomento à pesquisa: contribuição para uma estratégia de gestão da difusão para o CNPq. 2013. 161 f. Dissertação (Mestrado em Desenvolvimento Sustentável) - Universidade de Brasília, Brasília, 2013.

STORER, N. W. The social system of science. Nova York: Holt, Rinehart \& Winston, c1966. $180 \mathrm{p}$.

THE WELLCOME TRUST. Costs and business models in scientific research publishing: a report commissioned by the Wellcome Trust. London: SQW, 2004. Disponível em:

http://www.wellcome.ac.uk/stellent/groups/corporatesite/@policy_communicatio ns/documents/web_document/wtd003184.pdf. Acesso em: 25 nov. 2015.

WEITZEL, S. da R.; MACHADO, E. C. Estratégias para implementação de repositórios da UNIRIO. In: SEMINÁRIO NACIONAL DE BIBLIOTECAS UNIVERSITÁRIAS, 16., 2010, Rio de Janeiro. Anais eletrônicos... Rio de 
Janeiro: UFRJ, 2010. Disponível em: http://www.sibi.ufrj.br/snbu2010/pdfs/orais/final_197.pdf. Acesso em: 05 nov. 2015.

\title{
ACTORS OF THE SCHOLARLY COMMUNICATION SYSTEM: NOTES FOR DISCUSSION OF THEIR ROLES
}

\begin{abstract}
Introduction: The scholarly communication system, which underlies the scientific community, consists of elements and processes, between the positions they perform and the functions and activities. It is the result of the functions and activities of researchers, publishers, libraries, funding agencies and universities that contribute to the production, sharing and knowledge use so that a science can be made viable. Objective: Identify the functions and activities performed by the main actors of the scholarly communication system (researchers, publishers, libraries, development agencies and universities). Methodology: A large bibliographic survey was carried out in Google Scholar, Web of Science and Scopus in the months of June and July 2015. The survey disregarded time limit and type of document. The search strategy was a combination of the terms "researchers", "publishers", "libraries", "funding agencies" and "universities" combined with the terms "scientific communication", "scientific information", "open access", "communication system". After the preliminary analysis of the texts retrieved and the selection of the relevant texts, a literature review was elaborated for the purpose of identifying functions and activities performed by the main actors of the scholarly communication system. The revision took the character of narrative and was structured from each of the actors. Results: The results are presented in the form of a narrative literature review in which the functions and activities performed by each of the actors of the scholarly communication system contemplated in the study were defined and identified. Finally, a diagram was drawn up to represent graphically both the functions and activities of the actors of the scholarly communication system and the interactions between them.
\end{abstract}

Descriptors: Scholarly communication system. Scholarly communication system actors. Scholarly communication functions.

\section{ACTORES DEL SISTEMA DE COMUNICACIÓN CIENTIIFICA: APUNTES PARA DISCUSIÓN DE SUS FUNCIONES}

\section{RESUMEN}

Introducción: El sistema de comunicación científica, que es subyacente a la comunidad científica, está constituido por diversos elementos y procesos, entre ellos sus actores y sus funciones y actividades. Es el resultado de las funciones y actividades investigadores, editoriales, bibliotecas, agencias de fomento y universidades que contribuyen a que la producción, el compartir y el uso del conocimiento de modo que la ciencia pueda viabilizarse. Objetivo: Identificar las funciones y actividades desempeñadas por los principales actores del sistema de comunicación científica 
(investigadores, editoriales, bibliotecas, agencias de fomento y universidades). Metodología: Se realizó un amplio análisis bibliográfico en Google Scholar, Web of Science y Scopus en los meses de junio y julio de 2015. El levantamiento desconsideró límite temporal y tipo de documento. La estrategia de búsqueda fue la combinación de los términos "investigadores", "editoriales", "bibliotecas", "agencias de fomento" y "universidades" combinadas con expresiones "comunicación científica", "información científica", "acceso abierto", "sistema de la comunicación" "comunicación de la ciencia". Además de las búsquedas se realizaron en portugués y en Inglés, se adoptaron términos sinónimos y flexión singular / plural. Después del análisis preliminar de los textos recuperados y selección de los textos relevantes, se elaboró una revisión de literatura orientada por el objetivo de identificar funciones y actividades desempeñadas por los principales actores del sistema de comunicación científica. La revisión asumió carácter de narrativa y fue estructurada a partir de cada uno de los actores. Resultados: Los resultados se presentan en forma de revisión de literatura narrativa en la que se definieron e identificaron funciones y actividades desempeñadas por cada uno de los actores del sistema de comunicación científica contemplados en el estudio. Por último, se elaboró un diagrama que representa gráficamente tanto las funciones y actividades de los actores del sistema de comunicación científica en cuanto a las interacciones entre ellos. Se asumió carácter de narrativa y fue estructurada a partir de cada uno de los actores.

Descriptores: Sistema de comunicación científica. Actores del sistema de comunicación científica. Funciones de la comunicación científica. 